\title{
Dielectric breakdown characteristics of a high current metal vapor plasma
}

\author{
M. TISACK and R. SACKS* \\ Department of Chemistry, University of Michigan, Ann Arbor, MI 48109, U.S.A.
}

(Received 6 January 1987)

\begin{abstract}
Time- and spatially-resolved spectroscopy is used to study the early-time spectral features of the plasmas produced by high-current, capacitive discharges through thin silver films. Spectra are compared for several support gases including $\mathrm{CO}_{2}$, $\mathrm{He}$, and an $\mathrm{Ar} / \mathrm{O}_{2}$ mixture. All measurements were made during the first $40 \mu \mathrm{s}$ of the discharge. At atmospheric pressure for all three gases, spectra from support gas species show intense lines for only a brief interval between 10 and $30 \mu$ s after the start of the discharge. Greatest intensity from silver lines always occurs at the film surface; while greatest intensity from support gas species occurs about $2.0 \mathrm{~mm}$ from the film surface. A magnetic field of a few $\mathrm{kG}$ normal to the electric field in the plasma and parallel to the thin film surface almost completely eliminates spectral lines from the support gas species.
\end{abstract}

\section{INTRODUCTION}

THE HIGH-temperature metal vapor plasmas produced by the capacitive discharge vaporization of thin metal films have been used for the determination of trace metallic elements in both solution $[1,2]$ and solid powder [3-6] samples. High plasma temperature and density [7] result in rapid sample atomization and relative freedom from concomitant and sample particle size effects. Relative errors of 3-10\% are typical for the direct determination of some metallic elements at ppm concentrations in $500 \mu \mathrm{g}$ powder samples using aqueous solution calibration standards.

In most cases, thin films of $\mathrm{Ag}$ or Au are prepared on plastic substrates, and a solid powder or solution residue sample is placed on the film surface. The film is vaporized at atmospheric pressure by a capacitive discharge in the $0.5-1.0 \mathrm{~kJ}$ range. Dielectric breakdown of the metal vapor or the surrouding gas results in the formation of a highly luminous, transient plasma with a duration in the 1-10 ms range. The sample is atomized, excited, and to some extent ionized by interaction with the hot plasma gases.

The analytical performance of these plasmas is limited by the high discharge current, which causes a high rate of plasma expansion. This results in poorer sample-plasma interaction and rapid loss of sample vapor.

Recent studies have shown that the presence of an external magnetic field of a few kG or less can drastically alter important plasma properties including size and shape [8], spatial and temporal intensity distributions from atomic species [9], and sampling and analytical characteristics [10]. These magnetically tailored plasmas have been used for the direct determination of several metallic elements in solid powder samples [6].

The external magnetic field is generated by the oscillatory, underdamped plasma current in a large coil surrounding the plasma chamber. The magnetic field is oriented normal to the electric field in the plasma and parallel to the thin film surface so that the $\mathbf{E} \times \mathbf{B}(E$-cross- $B)$

*Corresponding author.

[1] D. V. Duchane and R. D. SACKS, Anal. Chem. 50, 1765 (1978).

[2] S. W. BREWER and R. D. SACKS, Anal. Chem. 57, 724 (1985).

[3] E. M. Clark and R. D. SACKS, Spectrochim. Acta 35B, 471 (1980).

[4] J. GoldBerG and R. SACKS, Anal. Chem. 54, 2179 (1982).

[5] J. SWAN and R. SACKS, Anal. Chem. 57, 1261 (1985).

[6] D. AlbERS and R. SACKS, Anal. Chem. 59, 593 (1987).

[7] S. Y. SUH and R. D. SACKs, Spectrochim. Acta 368, 1081 (1981).

[8] D. Albers, E. Johnson, M. Tisack and R. SAcks, Appl. Spectrosc. 40, 60 (1986).

[9] D. Albers and R. SACKS, Spectrochim. Acta 41 B, 968 (1986).

[10] D. Albers, M. TiSACK and R. SACKS, Appl. Spectrosc. 61, 131 (1987). 
drift motion $[11,12]$ of ions and electrons in the plasma is in the direction of the substrate surface. This results in greater sample-plasma interaction and a nearly order-of-magnitude increase in the rate of sample atomization. Shot-to-shot intensity reproducibility also may improve significantly.

In the present study, time- and spatially-resolved emission spectroscopy is used to study the early-time behavior of these analytically useful metal vapor plasmas and to show that the external magnetic field also can alter the dielectric breakdown characteristics of the metal vapor and the plasma support gas. This provides additional experimental control of the plasma formation processes, and may be exploited to further improve the analytical characteristics of the plasmas.

\section{Apparatus and Experiment Design}

\subsection{The $\mathbf{E} \times \mathbf{B}$ drift configuration}

In the presence of an external magnetic field, the linear momentum of ions and electrons in the direction normal to the magnetic field is converted to angular momentum, and the ions and electrons are guided by the magnetic field in circular paths in the plane normal to the field direction. The centers of these circular orbits are known as guiding centers, and they can move across field lines only during collisions or by the application of an additional force field with a component normal to the external magnetic field.

For the studies reported here, the electric field $\mathbf{E}$ in the plasma is oriented normal to the external magnetic field $\mathbf{B}$, with the result that an $\mathbf{E} \times \mathbf{B}$ drift motion of the guiding centers occurs in the direction normal to the plane containing the $\mathbf{E}$ and $\mathbf{B}$ vectors. This is discussed in more detail in Ref. [8]. The magnetic field is applied parallel to the surface of the plastic substrate which supports the thin film and sample prior to the discharge. This results in the $\mathbf{E} \times \mathbf{B}$ drift motion being directed normal to the substrate surface. With the magnetic field present and the drift motion directed toward the substrate surface, the plasma appears to be kept in close contact with the substrate during the entire duration of the discharge [8]. Without the magnetic field, or with the field direction reversed so that the drift motion is directed away from the substrate, the plasma rapidly leaves the substrate surface, and the interaction of the sample with the plasma is much poorer.

\subsection{Capacitive discharge apparatus}

In previous studies $[9,10]$, the coil used to generate the magnetic field was connected in series with the plasma, and thus the plasma current was also used to generate the magnetic field. This is necessary for analytical applications since the RCL tank circuit is underdamped, and the oscillating plasma current results in a $180^{\circ}$ change in the plasma electric field direction for every consecutive discharge current halfcycle. If the magnetic field is generated by the plasma current, the magnetic field direction also will rotate by $180^{\circ}$ for every half-cycle, and the direction of the $\mathbf{E} \times \mathbf{B}$ drift motion will be constant during the entire duration of the discharge. In addition, this arrangement is very convenient since only one capacitive discharge power supply is required.

This arrangement, however, was less satisfactory for some of the work reported here. This is because the discharge current is quite low during the formative stages of plasma development early in the first current half-cycle. This results in a relatively weak magnetic field which has only a modest effect on the dielectric breakdown processes occurring near the thin film surface. For some studies, a dual discharge system was developed where a separate RCL tank circuit was used to generate the magnetic field in the coil surrounding the plasma chamber. With the dual discharge system, a magnetic field of several kG was available during the very early stages of plasma formation.

A simplified diagram of the dual discharge circuit is shown in Fig. 1. Each discharge loop contains a $30 \mu \mathrm{F}$ capacitor which typically is charged to $5-10 \mathrm{kV}$. Circuit inductance is varied as needed in the range $100-1700 \mu \mathrm{H}$. Each circuit is fired on command by triggering a high current ignitron switch. The ignitron trigger circuit has been described [13]. Construction details of the coil used to generate the magnetic field $\left(\mathrm{L}_{\mathrm{M}}\right)$ are given in Ref. [8].

In typical operation, the discharge circuit producing the magnetic field contains much higher inductance and is triggered first. The circuit generaring the plasma is triggered at about the time of the first current peak of the field-producing circuit. Since the magnetic field is generated by a circuit containing much larger inductance, the oscillation frequency of the magnetic field is much lower than

[11] F F. Chen, Introduction to Plasma Physics and Controlled Fusion, 2nd Edn, Vol. 1. Plenum, New York (1984).

[12] T. J. M. Boyd and J. J. SANDERson, Plasma Dynamics. Barnes \& Noble, New York (1969).

[13] S. Y. SuH, R. J. Collins and R. D. SACKS, Appl. Spectrosc. 35, 42 (1981). 


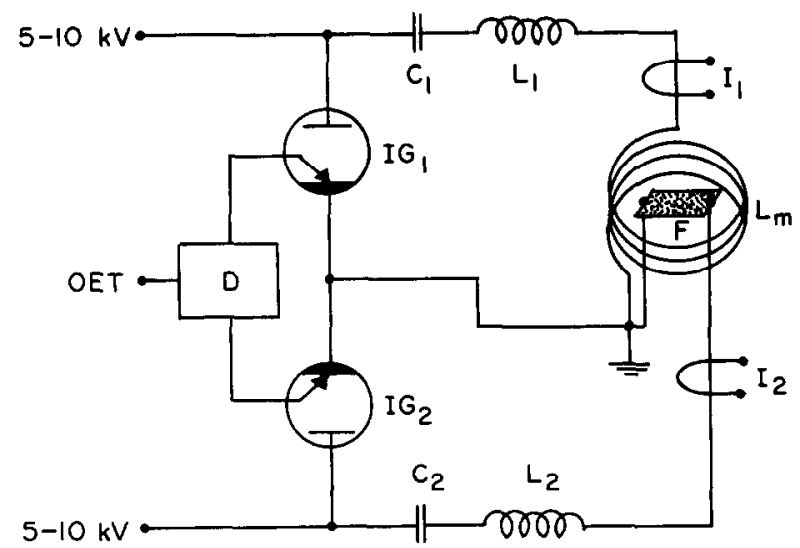

Fig. 1. Dual discharge circuit. $C_{1}$ and $C_{2}$, main discharge capacitor banks; $L_{1}$ and $L_{2}$, air-core inductors for adjusting ringing frequencies; $I G_{1}$ and $I G_{2}$, welding ignitron switches; $I_{1}$ and $I_{2}$, current monitoring transducers; $\mathrm{OET}$, optoelectronic triggering network; $\mathrm{D}$, time-delay network; $\mathrm{F}$, thin $\mathrm{Ag}$ film; $L_{M}$, magnetic-field coil.

the oscillation frequency of the plasma, and the magnetic field is fairly constant for the entire first current half-cycle of the plasma.

\subsection{Time-gating, spatially-resolving optical system}

A rotating-mirror streaking camera system similar to one described by SACKs and W ALTERs [14] and Sun and SACKS [7] was used to obtain time-gated, spatially-resolved photographic spectra. The thin film is oriented so that the electric field in the plasma is in the horizontal plane and normal to the optical axis. The coil producing the magnetic field is oriented with its axis along the optical axis. A pair of spherical mirrors in an over-and-under configuration is used to form an image of the plasma on a vertical timegate slit. A second pair of spherical mirrors, one of which is rotated at $60 \mathrm{~Hz}$ by a hysteresis synchronous motor, sweeps the image of the time-gate slit across the vertical spectrometer slit.

Astigmatic imaging is used to obtain spatial resolution of about $0.1 \mathrm{~mm}$ in the direction of the drift motion. Note that this optical system results in some spatial integration of radiation parallel to the electric field axis. However, since variations in plasma properties are relatively small in this direction, some spatial integration is useful in improving shot-to-shot reproducibility. Details of astigmatic imaging are found elsewhere [15]. The lateral magnification of the complete system is about 0.43 . The spectrometer entrance slit width was fixed at $100 \mu \mathrm{m}$, and the width of the time-gate slit was adjusted to give adequate exposure on the high speed emulsion from a single shot. Time gate width typically is $2.5 \mu \mathrm{s}$. The time gate width and location relative to the discharge current waveform are monitored for every shot by a photomultiplier tube placed inside the spectrometer. This tube responds to stray light in the instrument.

The rotating-mirror streaking camera also is used to generate the timing pulse needed to synchronize the angular position of the rotating mirror with the initiation of the discharges. The mirror sweeps the image of a small tungsten-filament bulb across a photodiode. The output pulse from the diode is shaped and used to start a timing circuit which delivers trigger pulses to the appropriate ignitron switches in the discharge circuit.

\subsection{Optical and electrical monitoring}

All spectra were obtained on a 1.0-m Czerny-Turner spectrometer (Jarrell-Ash Model 78-462) equipped with a $600 \mathrm{line} / \mathrm{mm}$ grating blazed for $500 \mathrm{~nm}$ in the first order. Spectra were recorded on Kodak Royal Pan-X film which was developed to obtain an ASA speed of about 2400 . This very coursegrain, high-fog film was required to obtain adequate response for single-shot exposures with an effective exposure time of about $2.5 \mu \mathrm{s}$. Spectra were recorded in the wavelength range $330-480 \mathrm{~nm}$. A $100 \mu \mathrm{m}$ spectrometer entrance slit was used in all cases. The time-gate slit typically was $300 \mu \mathrm{m}$ wide.

Microdensitometer traces of photographic spectra were obtained on a Joyce-Loebl Mark IIIB recording microdensitometer. For spatially-resolved spectra, the densitometer slit was $30 \mu \mathrm{m}$ wide and $100 \mu \mathrm{m}$ long. Considering the 0.43 value for lateral magnification of the complete optical system, the $100 \mu \mathrm{m}$ slit length limits spatial resolution in the plasma to about $0.23 \mathrm{~mm}$.

[14] R. D. SaCks and J. P. W alters, Anal. Chem. 42, 61 (1970).

[15] S. C. Salmon and J. A. Holcombe, Anal. Chem. 50, 1714 (1978). 
The discharge current was monitored with a wide-band, inductive current-to-voltage transducer (Pearson Electronics, Model 1025). The output from the transducer was displayed on a Nicolet Model 2090-III digital storage oscilloscope. This instrument has two nonmultiplexed, 12-bit input channels and a maximum sampling rate of $2.0 \mathrm{MHz}$. The output from the photomultiplier tube inside the spectrometer was monitored simultaneously with the discharge current waveform.

\subsection{Procedures and materials}

Silver thin films were prepared on polypropylene substrates as described previously [4]. Table 1 gives thin film/substrate properties and discharge conditions. Films were installed in a cassette which provides electrical contact to the film and contains a plasma confinement cell. The cell mechanically confines the plasma in the direction parallel to the electric field but provides no confinement parallel to the magnetic field axis (optical axis). This is described elsewhere in more detail [8]. Experiments were conducted at atmospheric pressure in $\mathrm{CO}_{2} \mathrm{He}$ and a $60 \% \mathrm{Ar} / 40 \% \mathrm{O}_{2}$ mixture.

\section{Results AND Discussion}

Both parts of the dual discharge circuit are underdamped, and thus produce damped, oscillatory discharge currents. Ringing frequencies are given in Table 1. Figure 2 shows discharge current waveforms from both discharge circuits and the voltage waveform from the

Table 1. Thin film/substrate properties and discharge conditions

\begin{tabular}{lcc}
\hline Thin Ag films & & \\
\hline Substrate material & Polypropylene & \\
Substrate dimensions $(\mathrm{cm})$ & $7.3 \times 1.6 \times 0.05$ & \\
Film thickness $(\mathrm{nm})$ & 40 & \\
Film area vaporized $\left(\mathrm{cm}^{2}\right)$ & 7.9 & Magnetic field \\
Film mass vaporized $(\mu \mathrm{g})$ & 350 & 7.0 \\
Film resistance $(\Omega)$ & $3.0-9.0$ & 1805 \\
\hline Discharge conditions & Plasma & 30 \\
\hline Charging voltage $(\mathrm{kV})$ & 6.0 & 735 \\
Inductance $(\mu \mathrm{H})$ & 105 & 0.68 \\
Capacitance $(\mu \mathrm{F})$ & 30 & 5.1 \\
Discharge energy $(\mathrm{J})$ & 540 & - \\
Ringing frequency $(\mathrm{kHz})$ & 2.8 & - \\
Peak magnetic field $(\mathrm{kG})$ & - & $\mathrm{O}$ \\
Plasma gas & $60 \% \mathrm{Ar} / 40 \% \mathrm{CO}_{2}$ and $\mathrm{He}$ & Atmospheric \\
Pressure & & \\
\hline
\end{tabular}

(a)

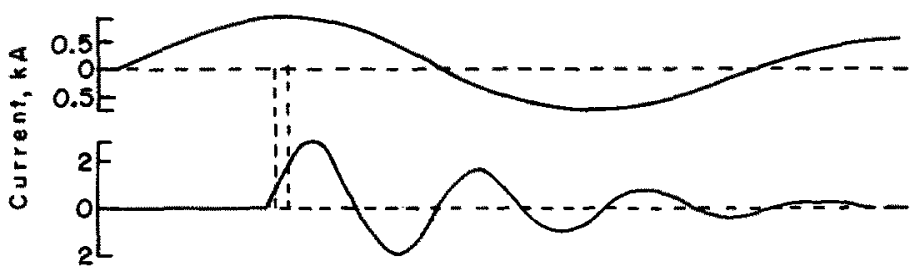

(b)

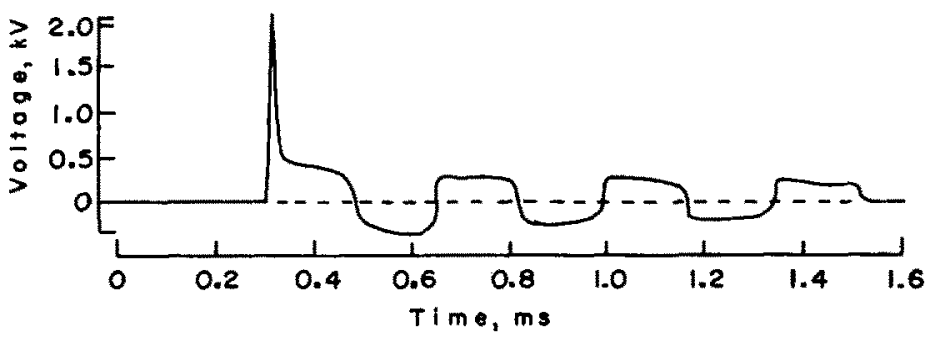

Fig. 2. Discharge current in the magnetic field coil (a), discharge current in the plasma (b), and voltage waveform across plasma (c). Broken vertical lines indicate the time interval of interest in this study. 
plasma. The discharge used to produce the magnetic field (a) is initiated about $0.3 \mathrm{~ms}$ before the discharge used to generate the plasma (b). This results in the peak value of magnetic field strength occurring during the time interval under investigation. This time interval is indicated by the pair of broken vertical lines. During this interval, the magnetic field strength changes by less than $1 \%$ from the nominal value of $5.1 \mathrm{kG}$. Note that the direction of the $\mathbf{E} \times \mathbf{B}$ drift motion changes direction by $180^{\circ}$ at the start of the second plasma current half-cycle, but this is of no concern for the studies reported here since all radiation measurements are completed long before this time.

The plasma current lasts for about $1.2 \mathrm{~ms}$ and reaches a peak value of about $2.5 \mathrm{kA}$ during the first current half-cycle. This particular current waveform was used for the direct determination of metallic elements in solid, powder samples by exploiting the $\mathbf{E} \times \mathbf{B}$ drift motion of the plasma to improve sample-plasma interaction [6]. During most of the discharge, the voltage is in the range $0.3-0.5 \mathrm{kV}$. However, very early in the first half-cycle of the discharge, a large spike is observed in the waveform.

Figure 3 shows an expanded view of the plasma current and voltage waveforms during the time interval of greatest interest in this study. Time-gated, spatially-resolved photographic spectra were obtained with time gates centered at $10,20,30$ and $40 \mu$ s after the start of the discharge. A typical output pulse from the photomultiplier tube monitoring stray radiation inside the spectrometer is shown in part (c) of Fig. 2 to provide a reference of time gate width relative to changes in the plasma current and voltage. The shape of the pulse from the photomultiplier tube is determined in part by the relative widths of the spectrometer slit and the external time slit.

The plasma current increases very rapidly, and is nearly $300 \mathrm{~A}$ by the time of the earliest time gate at $10 \mu \mathrm{s}$. A very slight discontinuity occurs in the current waveform at about $8 \mu \mathrm{s}$ after the start of the discharge. This may mark the occurrence of dielectric breakdown in the metal vapor or surrounding gas and the onset of gas-phase current conduction. No line radiation is observed prior to this time. The earliest time gate location at $10 \mu$ s represents the

(a)

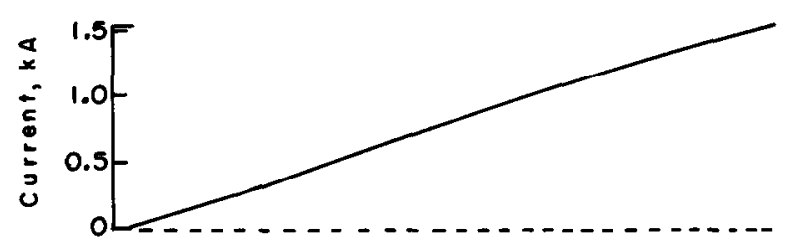

(b)

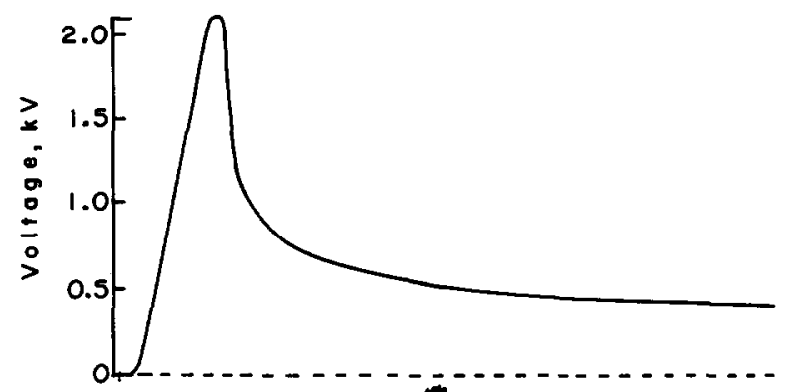

(c)

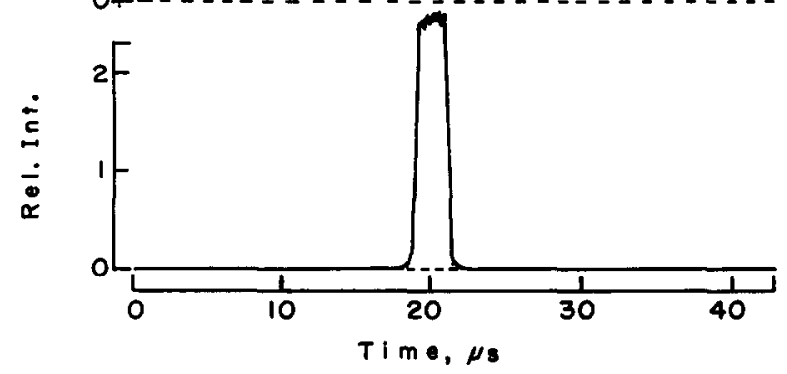

Fig. 3. Plasma current (a) and voltage (b) waveforms for the first $40 \mu \mathrm{s}$ of the discharge and output pulse (c) from the photomultiplier tube inside the spectrometer. 
earliest time for which a time-gate monitor pulse could be detected by the photomultiplier tube.

The voltage drop across the thin film/plasma increases almost linearly with time for about the first $5 \mu$ s of the discharge, and reaches a peak value of about $2.1 \mathrm{kV}$ at $6.5 \mu \mathrm{s}$. This feature is quite reproducible from shot to shot. Next, the voltage drop decreases very rapidly as the resistance of the plasma gases falls during dielectric breakdown. The dielectric breakdown processes must be quite different than those in a high voltage spark $[16,17]$ since the $2.1 \mathrm{kV}$ peak voltage observed in these experiments would not result in breakdown through the quiescent plasma gas. Dielectric breakdown at relatively low voltages may be caused by heating of the gas by a strong shock wave generated from the rapid vaporization of the thin film $[18,19]$ or by ionization of the gas from collisions with electrons thermionically ejected by the hot solid or liquid metal $[20,21]$. More detailed information during the time interval from $5-10 \mu$ s would be useful but will require a measurement system with a minimum sampling interval of less than $0.5 \mu \mathrm{s}$.

\subsection{Temporal features of the dielectric breakdown spectra}

Figure 4 shows microdensitometer traces from time-gated, spatially-integrated spectra of $\mathrm{Ag}$ thin films vaporized in a $60 \% \mathrm{Ar} / 40 \% \mathrm{O}_{2}$ gas mixture. Spectra are presented for time gates centered at 10,20 and $30 \mu$ s after the start of the discharge. No magnetic field was present. A number of prominent emission lines are labeled with an element symbol and a number. Silver lines from the thin film material are identified in Table 2; lines from atmospheric species are identified in Table 3 . Note the high noise level in the traces. This is the result of the very grainy nature of the high-speed emulsion.

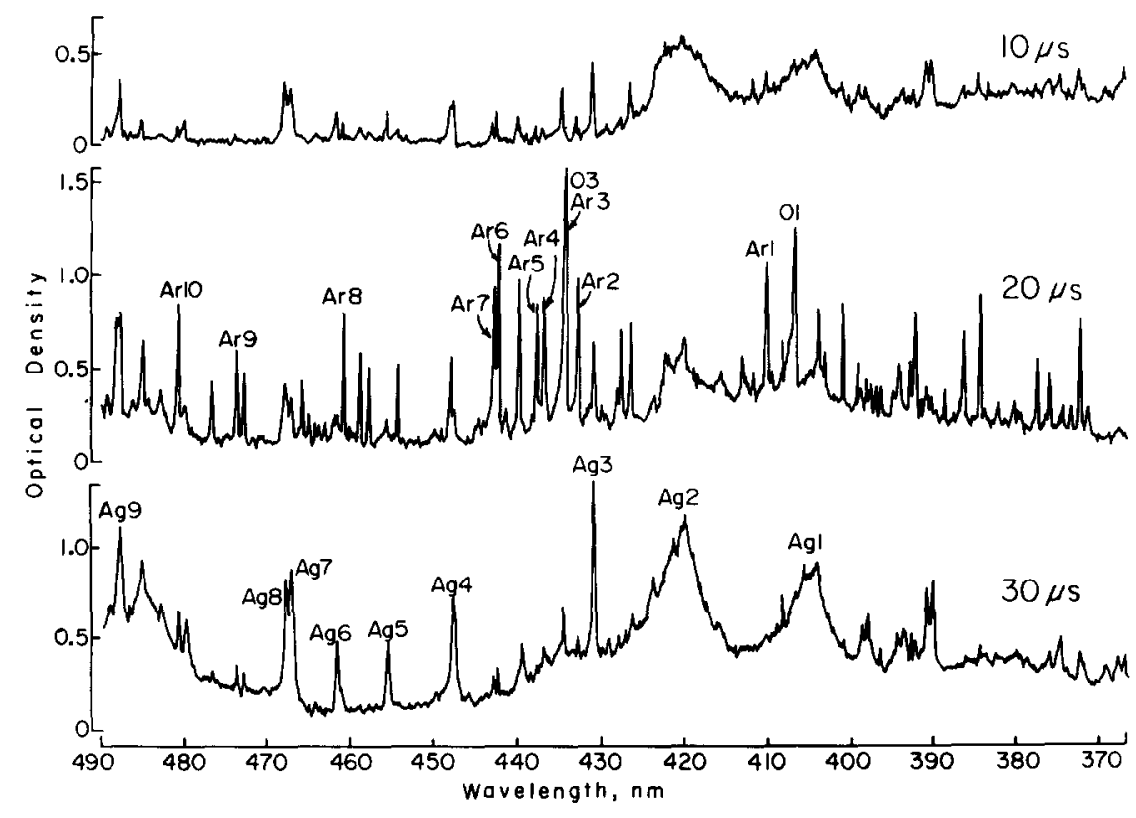

Fig. 4. Microdensitometer traces for time-gated, spatially-integrated spectra in a $60 \% \mathrm{Ar} / 40 \% \mathrm{O}_{2}$ gas mixture. Top, $10 \mu \mathrm{s}$ after start of discharge; center, $20 \mu \mathrm{s}$ after start of discharge; bottom, $30 \mu \mathrm{s}$ after start of discharge. See Tables 2 and 3 for line assignments.

[16] J. P. WALTERS, Appl. Spectrosc. 23, 317 (1969).

[17] A. von ENGEL, Ionized Gases, 2nd Edn. Oxford Press, London (1965).

[18] D. Y. Cheng, W. J. Loubsky and V. E. Fousekis, Phys. Fluids 14, 2328 (1971).

[19] F. D. Bennett, H. S. Burden and D. D. Shear, Phys. Fluids 5, 102 (1962).

[20] W. G. Chace, R. L. Morganand K. R. SaAri, Exploding Wires, Ed. W. G. Chace and H. K. Moore, Vol. 1, p. 59. Plenum Press, New York (1959).

[21] G. J. Woffinden, Exploding Wires, Ed. W. G. Chace and K. MoOre, Vol. 3, p. 193. Plenum Press, New York (1964). 
Table 2. Silver line assignments for Figs 4-6

\begin{tabular}{lcc}
\hline Designation & Species & Wavelength (nm) \\
\hline Ag1 & Ag I & 405.53 \\
Ag2 & Ag I & 421.09 \\
Ag3 & Ag I & 431.11 \\
Ag4 & Ag I & 447.60 \\
Ag5 & Ag I & 455.60 \\
Ag6 & Ag I & 461.57 \\
Ag7 & Ag I & 466.85 \\
Ag8 & Ag I & 467.79 \\
Ag9 & Ag I & 487.42 \\
\hline
\end{tabular}

Table 3. Support gas line assignments for Figs $4-6$

\begin{tabular}{lcc}
\hline Designation & Species & Wavelength (nm) \\
\hline Ar1 & Ar II & 410.39 \\
Ar2 & Ar II & 433.21 \\
Ar3 & Ar II & 437.14 \\
Ar4 & Ar II & 437.94 \\
Ar5 & Ar II & 442.60 \\
Ar6 & Ar II & 443.02 \\
Ar7 & Ar II & 460.96 \\
Ar8 & Ar II & 473.59 \\
Ar9 & Ar II & 480.61 \\
O1 & O II & 407.58 \\
O2 & O II & 411.92 \\
O3 & O II & 434.94 \\
O4 & O II & 441.49 \\
O5 & O II & 464.18 \\
O6 & O II & 464.92 \\
C1 & C II & 387.62 \\
C2 & C II & 392.07 \\
C3 & C II & 426.73 \\
He1 & He I & 388.87 \\
He2 & He I & 402.62 \\
\hline
\end{tabular}

At $10 \mu \mathrm{s}$, the strongest spectral features are from the thin film material. The neutral-atom $\mathrm{Ag}$ lines at 405.6 and $421.1 \mathrm{~nm}$ (lines marked $\mathrm{Ag} 1$ and $\mathrm{Ag} 2$ ) are extremely broad, and they contribute considerable background radiation at wavelengths less than about $430 \mathrm{~nm}$. A few Ar ion lines also are observed but at lower intensity.

The 20- $\mu$ s spectrum is drastically different and considerably more complex than the 10- $\mu \mathrm{s}$ spectrum. The Ar ion lines are very intense and dominate the spectrum. A few oxygen ion lines also are observed. Most of the Ag lines appear somewhat less intense than at $10 \mu \mathrm{s}$. While most of the Ar ion lines are several $\AA$ wide, they are usually narrower than the $\mathbf{A g}$ neutral-atom lines.

The $30-\mu$ s spectrum is drastically different from the $20-\mu$ s spectrum. All spectral features from the support gas species have virtually disappeared, and the spectrum again is dominated by $\mathrm{Ag}$ neutral-atom lines. However, the lines are much more intense than in the $10-\mu \mathrm{s}$ spectrum. Continuum background now is detected at all recorded wavelengths, and a highly broadened composite of $\mathrm{Ag}$ lines has become an important feature in the $480-490 \mathrm{~nm}$ region.

Figure 5 shows a set of microdensitometer traces obtained under similar conditions except that the plasmas were generated in a $\mathrm{CO}_{2}$ atmosphere. Again, no magnetic field was present. Some prominent lines are identified in Tables 2 and 3. The qualitative trends observed with increasing time are strikingly comparable to those of Fig. 4. At $10 \mu \mathrm{s}$ after the start of the discharge, the spectrum again is dominated by the very broad Ag neutral-atom lines at 421.1 and $405.6 \mathrm{~nm}$. These lines are somewhat more intense in $\mathrm{CO}_{2}$ than in the $\mathrm{Ar} / \mathrm{O}_{2}$ atmosphere. 


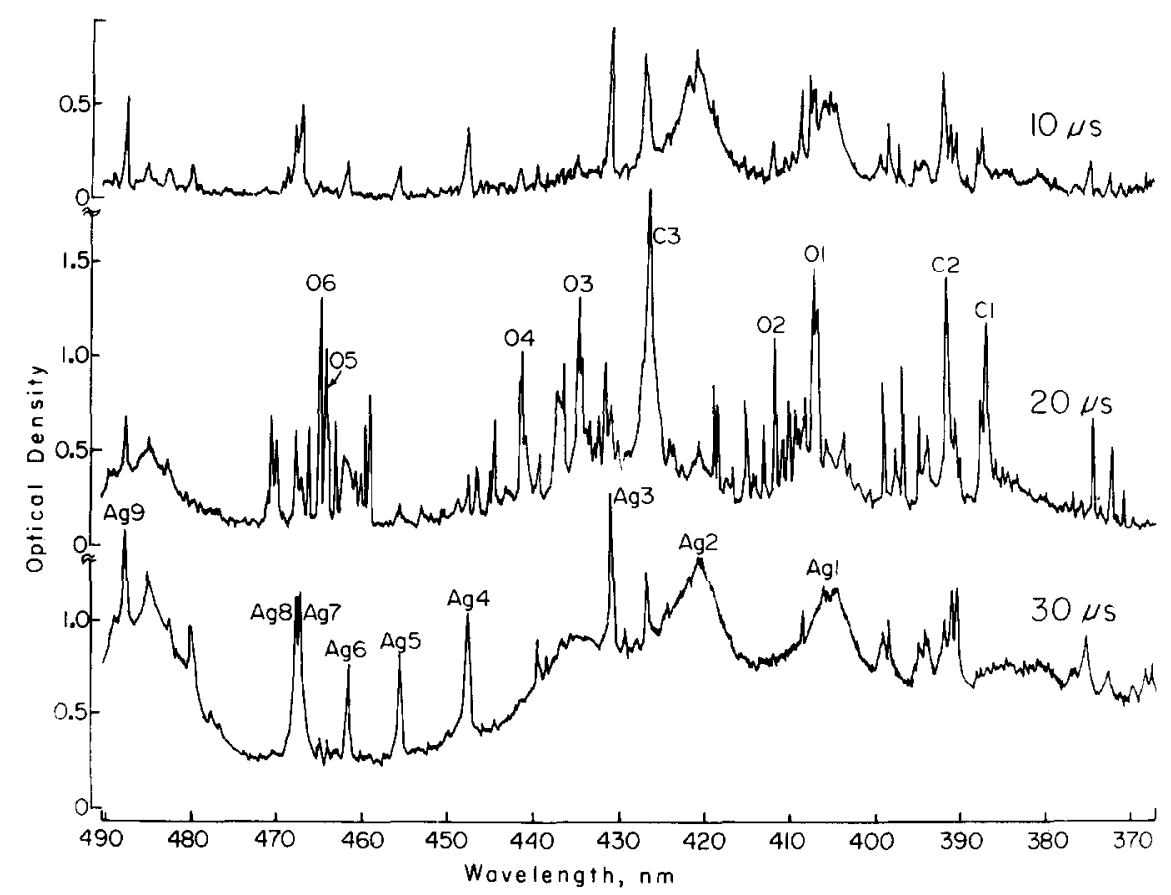

Fig. 5. Microdensitometer traces for time-gated, spatially-integrated spectra in a $\mathrm{CO}_{2}$ atmosphere. Top, $10 \mu \mathrm{s}$ after start of discharge; center, $20 \mu \mathrm{s}$ after start of discharge; bottom, $30 \mu \mathrm{s}$ after start of discharge. See Tables 2 and 3 for line assignments.

Also note the strong $\mathrm{C}$ ion line at $426.73 \mathrm{~nm}$. Apparently this originates from the decomposition of the $\mathrm{CO}_{2}$ gas. As in Fig. 4, the 20- $\mu$ s spectrum in Fig. 5 is dominated by lines from the support gas, and many of the $\mathrm{Ag}$ lines are actually lower in intensity than in the 10- $\mu \mathrm{s}$ spectrum. Note that the $426.73-\mathrm{nm} \mathrm{C}$ ion line is very intense and quite broad. A number of intense $\mathrm{O}$ ion lines also are observed throughout the wavelength region. At $30 \mu \mathrm{s}$, the spectrum again is dominated by $\mathrm{Ag}$ lines, and the qualitative features are quite similar to those in Fig. 4. While the 426.73-nm C ion line is clearly visible at $30 \mu \mathrm{s}$, its intensity is much lower than at $20 \mu \mathrm{s}$.

Figure 6 shows spectra obtained in an He atmosphere at the same three time gates. These spectra have far fewer lines, particularly at $20 \mu \mathrm{s}$. In addition, most lines are much narrower than in the other gases. This is most apparent for the lines labeled $\mathrm{Ag} 1$ and $\mathrm{Ag} 2$. This may be the result of lower metal vapor density near the substrate surface due to reduced inertial confinement in the lower density He environment.

At $10 \mu \mathrm{s}$, the strongest feature is the $\mathrm{He}$ (I) line at $388.87 \mathrm{~nm}(\mathrm{He} 1)$. The $402.62 \mathrm{~nm} \mathrm{He}$ neutral-atom line also is quite intense. No other He lines are observed for any time gate. Unlike the other gases, the strongest $\mathrm{Ag}$ I feature in $\mathrm{He}$ at $10 \mu \mathrm{s}$ is the $447.60 \mathrm{~nm}$ line, which is quite broad and self reversed. At $20 \mu \mathrm{s}$, a number of additional Ag lines appear. The $\mathrm{He}$ $388.87 \mathrm{~nm}$ line is almost the same intensity as at $10 \mu \mathrm{s}$. At $30 \mu \mathrm{s}$, the He lines are much weaker, and the spectrum is qualitatively similar to those in the other gases. However, continuum background intensity in $\mathrm{He}$ is much lower, and the $\mathrm{Ag} \mathrm{I}$ lines are significantly narrower than in the other gases.

The spectral features in Figs 4-6 strongly indicate that atmospheric species play an important role in the formative stages of plasma development. These data suggest that atmospheric species may be crucial to the dielectric breakdown process and to the transfer of current conduction from the solid or liqud $\mathrm{Ag}$ film to the $\mathrm{Ag}$ vapor. These atmospheric species may, in fact, be the principal carriers of the plasma current until current conduction transfer's back to the $\mathrm{Ag}$ vapor species between 20 and $30 \mu \mathrm{s}$ after the start of the discharge.

\subsection{Time-gated, spatially-resolved spectral features}

For the time-gated, spatially-integrated spectra in the $\mathrm{Ar} / \mathrm{O}_{2}$ and $\mathrm{CO}_{2}$ atmospheres, the transition from $20 \mu \mathrm{s}$ to $30 \mu$ s is probably the most interesting feature. Figures 7 and 8 show 


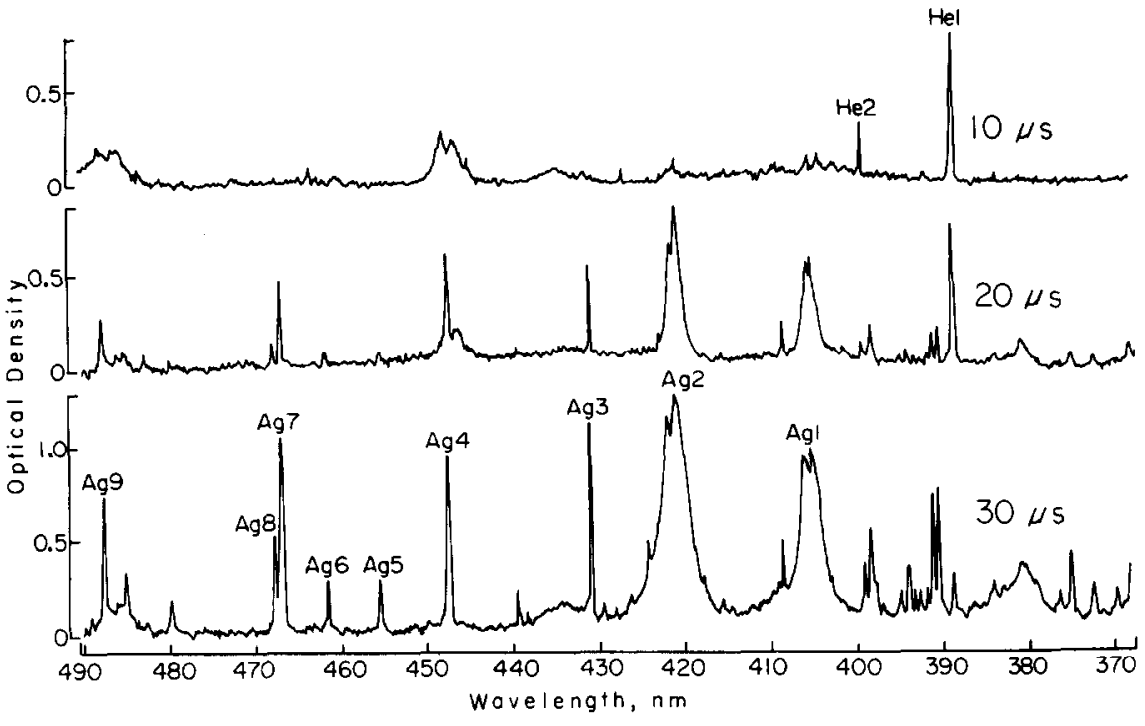

Fig. 6. Microdensitometer traces for time-gated, spatially-integrated spectra in a He atmosphere. Top, $10 \mu \mathrm{s}$ after start of discharge; center, $20 \mu \mathrm{s}$ after start of discharge; bottom, $30 \mu \mathrm{s}$ after start of discharge. See Tables 2 and 3 for line assignments.

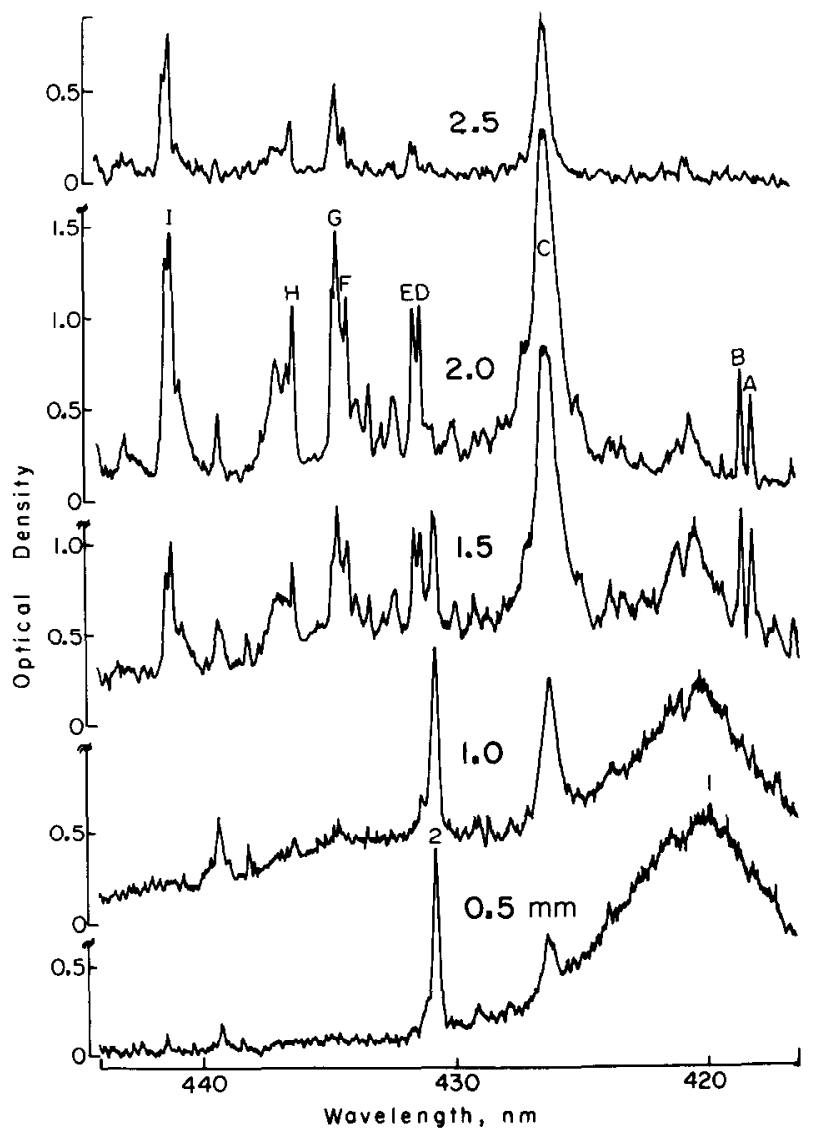

Fig. 7. Microdensitometer traces from time-gated, spatially-resolved photographic spectra at $20 \mu \mathrm{s}$ in a $\mathrm{CO}_{2}$ atmosphere. Traces were obtained at $0.5-\mathrm{mm}$ intervals from the substrate surface. See Table 4 for line assignments. 


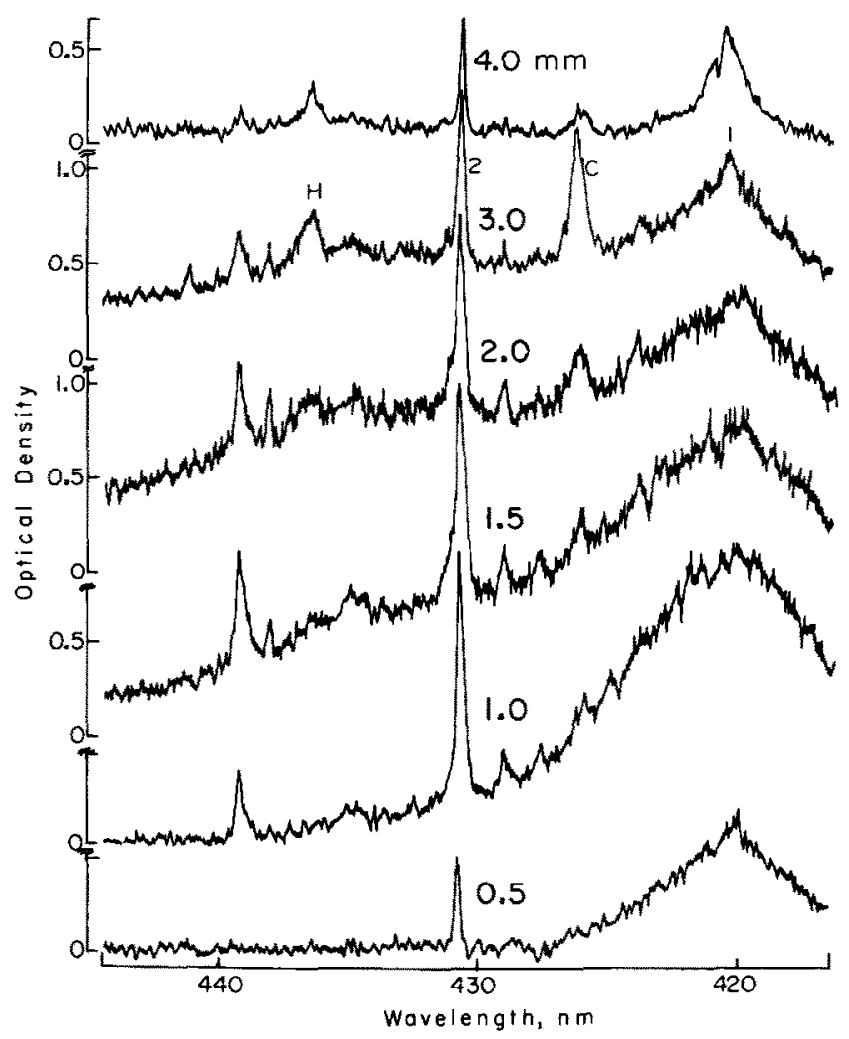

Fig. 8. Microdensitometer traces from time-gated, spatially-resolved photographic spectra at $30 \mu \mathrm{s}$ in a $\mathrm{CO}_{2}$ atmosphere. Traces were obtained at $0.5-\mathrm{mm}$ intervals from the substrate surface to $2.0 \mathrm{~mm}$ from the substrate and at $1.0-\mathrm{mm}$ intervals from 2.0 to $4.0 \mathrm{~mm}$ from the substrate. See Table 4 for line assignments.

Table 4. Line assignments for Figs 7 and 8

\begin{tabular}{lcc}
\hline Designation & Species & Wavelength (nm) \\
\hline I & $\mathrm{Ag} \mathrm{I}$ & 421.09 \\
2 & $\mathrm{Ag} \mathrm{I}$ & 431.11 \\
$\mathrm{~A}$ & O II & 418.55 \\
B & O II & 418.98 \\
C & C II & 426.73 \\
D & O II & 431.72 \\
E & O II & 431.97 \\
F & O II & 434.74 \\
G & O II & 434.94 \\
H & O II & 436.69 \\
I & O II & 441.49 \\
\hline
\end{tabular}

spatially-resolved microdensitometer traces from $20-\mu \mathrm{s}$ and $30-\mu$ s spectra, respectively, obtained in the $\mathrm{CO}_{2}$ atmosphere. For the 20- $\mu$ s spectrum, traces were obtained at $0.5-\mathrm{mm}$ intervals in the range from 0.5 to $2.5 \mathrm{~mm}$ from the substrate surface. For the $30-\mu \mathrm{s}$ spectrum, traces were obtained at $1.0-\mathrm{mm}$ intervals in the range from 2.0 to $4.0 \mathrm{~mm}$ above the substrate and at $0.5-\mathrm{mm}$ intervals closer to the substrate. Line assignments are found in Table 4.

At $0.5 \mathrm{~mm}$ from the substrate, the traces for the two time gates are quite similar except for somewhat greater intensity at $20 \mu$ s and the presence of a small peak from the $426.73-\mathrm{nm} \mathrm{C}$ ion line. For both time gates, the $421.09-\mathrm{nm} \mathrm{Ag}$ line is extremely broad. At $1.0 \mathrm{~mm}$ above the substrate surface, the spectra change relatively little except for somewhat greater intensity of the $\mathrm{Ag}$ lines at $30 \mu \mathrm{s}$, and of the $\mathrm{C}$ ion line at $20 \mu \mathrm{s}$. 
At $1.5 \mathrm{~mm}$ from the substrate, the spectra from the two time gates are drastically different. At $20 \mu \mathrm{s}$ the $\mathrm{C}$ ion line is very intense, and is the most prominent feature in the spectrum. In addition, the $\mathrm{Ag}$ line intensities are somewhat lower and the lines narrower than at distances closer to the substrate. A very important feature of the $1.5-\mathrm{mm}$ trace at $20 \mu \mathrm{s}$ is the appearance of numerous strong oxygen ion lines. These $\mathrm{O}$ lines and the $\mathrm{C}$ ion line are just barely detected over the emulsion grain noise in the $1.5-\mathrm{mm}$ trace at $30 \mu \mathrm{s}$.

At $2.0 \mathrm{~mm}$ from the substrate surface, the $20-\mu \mathrm{s}$ trace shows very intense $C$ and $O$ ion lines, but the Ag neutral-atom lines are greatly diminished in intensity. The $\mathrm{Ag} 421.09$ line is much narrower than nearer the substrate surface, and it is a relatively minor feature of the spectrum. For the $30-\mu$ s spectrum, the $\mathrm{C}$ and $\mathrm{O}$ ion lines again are just present over grain noise, and the $\mathrm{C}$ line is only slightly more intense. Perhaps the most significant feature in the $30-\mu$ s trace at $2.0 \mathrm{~mm}$ is the significantly higher continuum background intensity.

At $2.5 \mathrm{~mm}$ in the $20-\mu \mathrm{s}$ spectrum, the Ag lines are virtually gone, and the $\mathrm{O}$ and $\mathrm{C}$ lines have much lower intensities than at $2.0 \mathrm{~mm}$. At $3.0 \mathrm{~mm}$, the $20-\mu$ s trace (not shown in Fig. 7) shows only very small peaks for the $441.49-\mathrm{nm} O$ line and the $\mathrm{Cline}$. In the $30-\mu \mathrm{s}$ spectrum, the $3.0-$ $\mathrm{mm}$ scan shows the greatest intensity for the $\mathrm{O}$ and $\mathrm{C}$ ion lines. However, these intensites are very low relative to the maximum intensities observed in the $20-\mu \mathrm{s}$ spectrum. At $4.0 \mathrm{~mm}$ from the substrate, all species show diminished intensity, but the $\mathrm{Ag}$ lines are still considerably more intense than at $2.5-\mathrm{mm}$ above the substrate surface in the $20-\mu \mathrm{s}$ spectrum.

Time-gated, spatially-resolved spectra in the $60 \% \mathrm{Ar} / 40 \% \mathrm{O}_{2}$ mixture are closely analogous to the spectra in $\mathrm{CO}_{2}$ shown in Figs 7 and 8. These data suggest that current conduction in the plasma during the first $30 \mu$ s of the discharge is very localized and is primarily confined to a sheath extending no more than about $3 \mathrm{~mm}$ from the substrate surface. Dielectric breakdown of the plasma support gas just above the thin film appears to be the origin of the intense radiation from atmospheric species observed in this region. At atmospheric pressure, dielectric breakdown appears to occur between 6 and $8 \mu \mathrm{s}$ after the start of the discharge current when the voltage drop across the burgeoning plasma is a maximum. Dielectric breakdown seems to occur in all three of the plasma support gases investigated in this study. Note that under quiescent conditions, dielectric breakdown could not occur in any of the gases. While atmospheric species may be the principal current carriers early in the discharge, current conduction appears to transfer back to species from the thin film material between 20 and $30 \mu$ s after the start of the discharge.

\subsection{Magnetic field effects on the dielectric breakdown spectra}

Preliminary studies were made with a single discharge circuit where the current used to generate the plasma was also used to generate the magnetic field in the coil surrounding the plasma. In previous studies $[6,8-10]$, this technique was found to promote drastic changes in plasma properties which could be exploited to improve shot-to-shot reproducibility, dramatically increase the rate of sample atomization and improve analytical accuracy. However, this technique produced only minor changes in the early-time dielectric breakdown spectra. The discharge circuit used for these preliminary studies contained $105 \mu \mathrm{H}$ of inductance in the field-producing coil. With the discharge parameters of Table 1, a peak current in the first discharge half-cycle of about $2.5 \mathrm{kA}$, and a peak magnetic field strength of $3.7 \mathrm{kG}$ were obtained. However, at the time of dielectric breakdown, the discharge current is only about $0.25 \mathrm{kA}$ and the field strength is only about $0.37 \mathrm{kG}$. This is insufficient to significantly "magnetize" either ions or electrons at atmospheric pressure. Witn the dual discharge system developed for the present studies, a field strength of about $5.1 \mathrm{kG}$ is present at the time of dielectric breakdown and plasma formation.

Since continuum background radiation usually is associated with the current conducting species in the plasma, the temporal and spatial distribution of continuum radiation can provide useful information on plasma structure. Figure 9 shows microdensitometer traces of continuum radiation from plasmas generated in the $\mathrm{Ar} / \mathrm{O}_{2}$ atmosphere with the dualdischarge circuit. Scans were made normal to the wavelength axis in a line-free region near $452 \mathrm{~nm}$. These scans show the distribution of continuum background with respect to distance from the substrate surface. Data are shown for four time-gate values both with (solid lines) and without (broken lines) the $5.1 \mathrm{kG}$ magnetic field. 
(a)

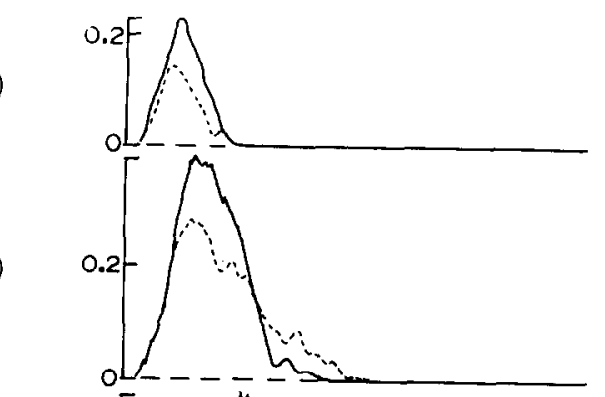

(b)

(d)

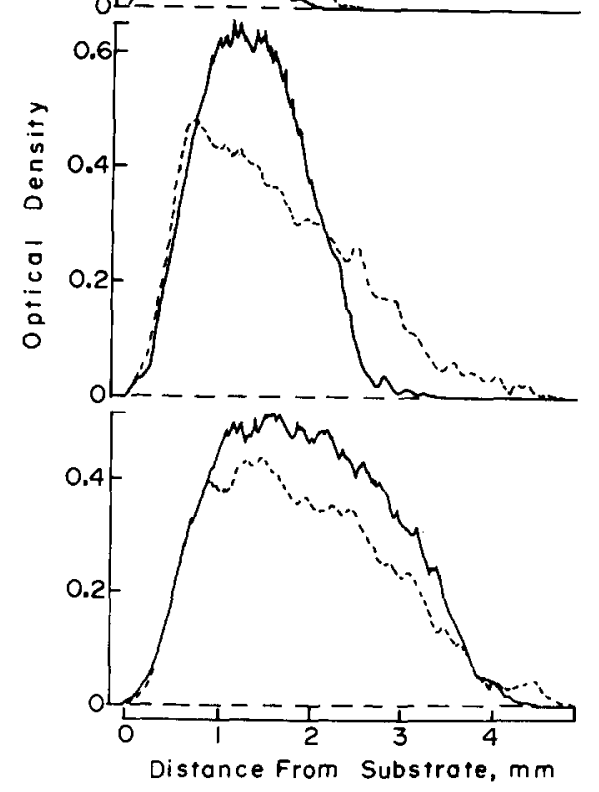

Fig. 9. Microdensitometer traces of continuum background radiation with no magnetic field (broken lines) and with a $5.1 \mathrm{kG}$ magnetic field (solid lines). Scans were made normal to the wavelength axis and reflect changes in continuum background intensity as a function of distance from the substrate surface. (a) $10 \mu \mathrm{s}$ after start of discharge; (b) $20 \mu \mathrm{s}$; (c) $30 \mu \mathrm{s}$; (d) $40 \mu \mathrm{s}$.

The shape of the traces in the region near the substrate surface, where intensity increases rapidly with distance, may be largely controlled by the change in solid angle with distance from the substrate surface. For the four time gates investigated, the peak continuum intensity is significantly higher with the field present. In addition, the leading edge of the advancing band of continuum radiation is steeper with the field present. This is most notable in traces (b) and (c) for time gates at 20 and $30 \mu \mathrm{s}$, respectively, after the start of the discharge. Note that $20 \mu \mathrm{s}$ is about the time of the most active participation of atmospheric species in the currentconduction processes.

Since higher continuum intensity may reflect higher current density in the plasma, the data in Fig. 9 suggest that the presence of the magnetic field results in a significant change in the shape of the current channel and in the peak current density. Previous studies [8] have shown that the $\mathbf{E} \times \mathbf{B}$ geometry used in this experimental system results in a drift motion of ions and electrons in the magnetically-tailored plasma. In the present study, this drift motion is toward the substrate surface and is probably responsible for the higher continuum intensity near the surface.

Figure 10 shows portions of time-gated, spatially-resolved photographic spectra obtained in the $\mathrm{Ar} / \mathrm{O}_{2}$ mixture at two time-gate locations. Spectrum (a) was obtained $20 \mu \mathrm{s}$ after the start of the discharge with no magnetic field present; while (b) was obtained at $20 \mu \mathrm{s}$ with the field present. Spectra (c) and (d) were obtained $30 \mu \mathrm{s}$ after the start of the discharge without and with, respectively, the $5.1 \mathrm{kG}$ field. Line assignments are provided in Table 5 .

The 20- $\mu$ s spectra with and without the field are drastically different. Without the field, a number of strong Ar ion lines are observed in a narrow zone extending from about 1 to $3 \mathrm{~mm}$ 
(a)

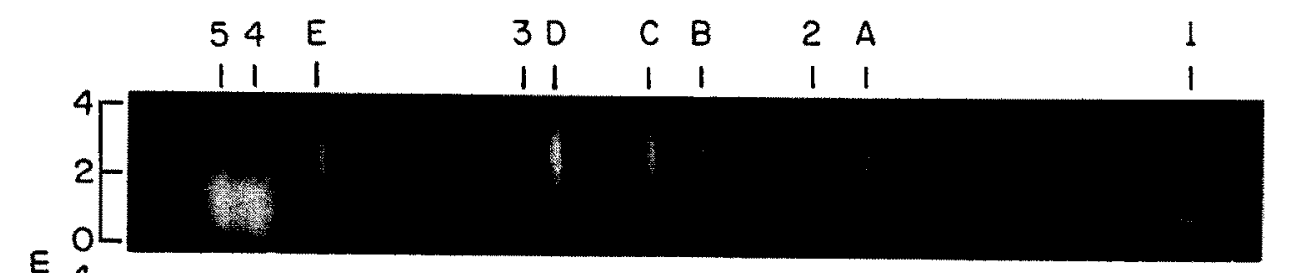

(b)

(c)

(d)
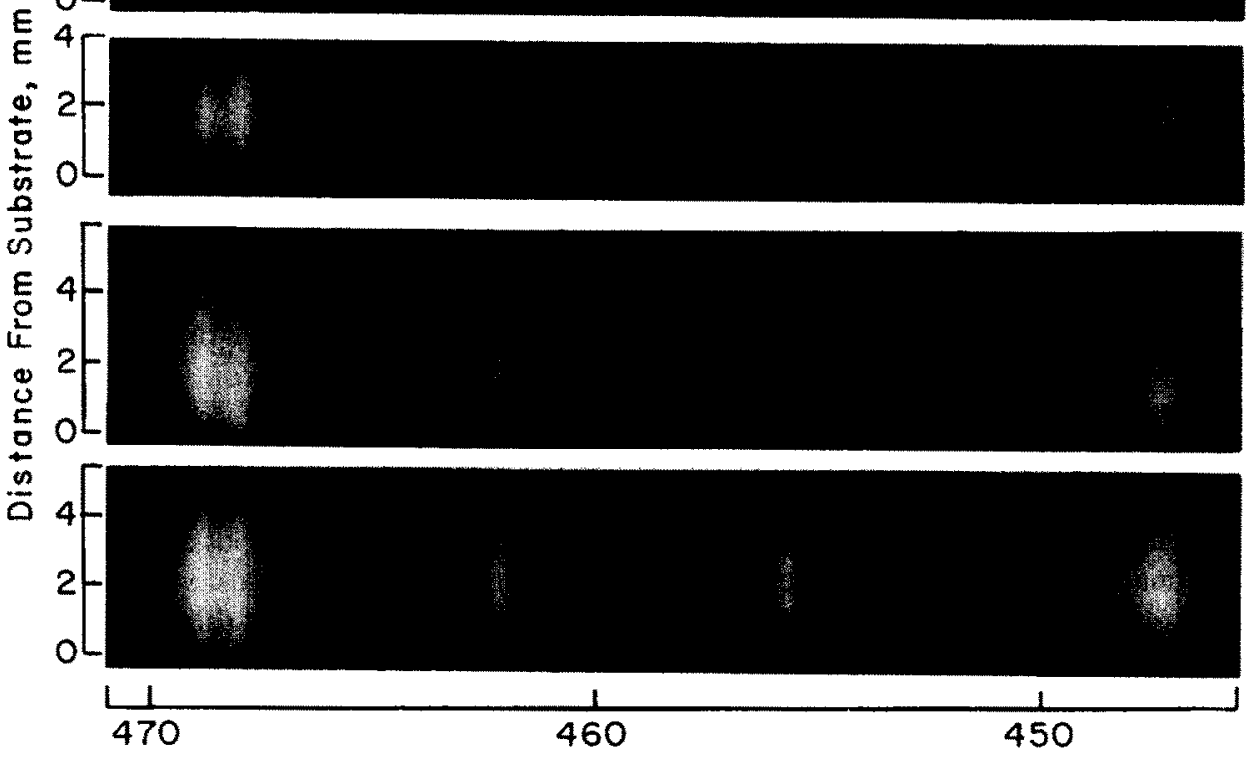

$$
\text { Wavelength, } \mathrm{nm}
$$

Fig. 10. Time-gated, spatially-resolved photographic spectra in an Ar- $\mathrm{O}_{2}$ mixture. (a) $20 \mu$ s after start of discharge and no magnetic field; (b) $20 \mu$ sith the magnetic field; (c) $30 \mu$ s with no magnetic field; (d) $30 \mu$ s with the magnetic field. See Table 5 for line assignments.

Table 5. Line assignments for Fig. 10

\begin{tabular}{lcc}
\hline Designation & Species & Wavelength (nm) \\
\hline 1 & $\mathrm{Ag} \mathrm{I}$ & 447.60 \\
2 & $\mathrm{Ag} \mathrm{I}$ & 455.60 \\
3 & $\mathrm{Ag} \mathrm{I}$ & 461.57 \\
4 & $\mathrm{Ag} \mathrm{I}$ & 466.85 \\
5 & $\mathrm{Ag} \mathrm{I}$ & 467.79 \\
$\mathrm{~A}$ & $\mathrm{Ar} \mathrm{II}$ & 454.51 \\
B & Ar II & 457.84 \\
C & Ar II & 458.99 \\
D & Ar II & 460.96 \\
E & Ar II & 465.79 \\
\hline
\end{tabular}

above the substrate surface. In addition, several $\mathrm{Ag}$ neutral-atom lines are observed. However, these lines are spatially separated from the Ar lines and show greatest intensity in the region extending from the substrate surface to about $1.5 \mathrm{~mm}$ above the substrate. Peak Ag intensity occurs about $0.7 \mathrm{~mm}$ above the substrate. Also note that most intense continuum background is found in the region from 1.0 to $2.0 \mathrm{~mm}$ above the substrate.

With the magnetic field present, the $\mathrm{Ar}$ ion lines are completely suppressed, and only $\mathrm{Ag}$ neutral-atom lines are observed. These lines are confined to the region from the substrate surface to about $3 \mathrm{~mm}$ above the substrate. Peak intensity occurs near the center of this region about $1.5 \mathrm{~mm}$ above the substrate. Both the $\mathrm{Ag}$ lines and the continuum background are significantly more intense with the field present.

At $30 \mu \mathrm{s}$ after the start of the discharge, the spectra with and without the magnetic field are more similar. This is largely because of the disappearance of most of the Ar ion lines in the 
spectrum obtained without the field. The most intense Ar lines are still observed but at very low intensity. Without the field, the $\mathrm{Ag}$ lines extend from the substrate to about $4 \mathrm{~mm}$ above the substrate, but the peak intensity still occurs about $0.7 \mathrm{~mm}$ from the substrate. Continuum background, however, is most intense about $2 \mathrm{~mm}$ above the substrate. With the field, the $\mathrm{Ag}$ lines again extend to about $4 \mathrm{~mm}$ above the substrate but this region appears more symmetric with greatest intensity from the $\mathrm{Ag}$ lines and the continuum background occurring about $2 \mathrm{~mm}$ above the substrate. As is the case for the $20-\mu \mathrm{s}$ spectra, the $30-\mu \mathrm{s}$ spectra show greater $\mathrm{Ag}$ line and background intensity with the field present.

\section{Conclusions}

It appears that without the magnetic field, dielectric breakdown of the atmosphere near the thin film surface occurs early in the discharge with the transfer of current conduction from the hot solid or liquid metal to ionic species of the atmosphere. Initial ionization of atmospheric species may occur at about the time of the peak voltage drop across the thin film. Collisional ionization of the gas by hot electrons in the tail of the electron-velocity distribution is more likely in the presence of a large electric field. The most likely source of electrons is from thermionic emission by the hot metal. Since the burgeoning plasma may have much lower resistance than the thin film, current and thus ohmic dissipation in the thin film may be greatly reduced after dielectric breakdown. This may retard vaporization of the thin-film residue. After dielectric breakdown of the gas, atomization of thin film material may proceed primarily by an indirect route involving ablation by the hot plasma gases.

The effect of the magnetic field on the early-time spectral features of the plasma provides considerable insight into the plasma formation processes. The lack of radiation from Ar ion species and the greater $\mathrm{Ag}$ radiation intensity with the magnetic field present suggest that dielectric breakdown does not occur in the gas, and more electrical energy is deposited directly in the thin film material. The $\mathbf{E} \times \mathbf{B}$ drift motion of thermionic electrons would keep them very near the thin film surface, and would thus reduce the probability of collisional ionization of the surrounding gas. This implies that greater electron kinetic temperature may be required to liberate electrons from the metal, and metallic-type current conduction may occur in the metal vapor for a longer period of time.

The analytical implications of a strong magnetic field present during the formative stages of the plasma are uncertain. Most previous analytical work with these metal-vapor plasmas has used time-gated intensity integration where radiation from the first current half-cycle of the discharge was not observed. Thus, the early-time spectral features from atmospheric species would not be a significant factor. However, the increased current conduction in the metal vapor near the substrate surface with the field present may increase the rate of sample atomization. In addition, improved shot-to-shot reproducibility may be achieved since earlier work [13] has shown that dielectric breakdown of the plasma support gas causes increased shot-to-shot variations in continuum background intensity. 